\title{
The effect of weight loss on obstructive sleep apnea (OSA) severity and position dependence in the bariatric population
}

\author{
Sophia E. Schiza • Charalampos Mermigkis • \\ Izolde Bouloukaki
}

Received: 18 February 2014 / Accepted: 3 March 2014 /Published online: 18 March 2014

(C) Springer-Verlag Berlin Heidelberg 2014

Obstructive sleep apnea (OSA) is a common chronic disorder, whose severity and duration predisposes to neurocognitive and medical sequelae development and varies among individuals. Obstructive respiratory events can be more severe and frequent in the supine sleeping position: indeed, more than half of all patients with obstructive sleep apnea (OSA) can be classified as supine-related OSA. The anatomical and physiological mechanisms for this phenomenon have not been well explained yet. In the supine posture, due to the unfavourable gravitational effects, the upper airway calibre could be reduced, resistance is likely to be increased, and therefore, the tendency for the upper airway to collapse is worsening compared to lateral position.

Since the first large study of Oksenberg et al. of 574 OSA patients, who found that $55.9 \%$ had positional OSA (POSA) and the prevalence was higher in mild to moderate OSA patients (ranging from 65-69\%) than in severe OSA [1], several studies with similar findings were published. These patients tend to have less severe OSA, to be less obese and to be younger [2]. Even in those patients in whom the apnea hypopnea index (AHI) is not influenced by body position, the duration of apnea/hypopnea and the degree of associated desaturations seems to be worse in the supine position [3].

Avoidance of the supine posture is efficacious and most studies report a positive effect of positional therapy (PT) on the AHI. Additionally, conservative treatment of OSA, including weight reduction, can be just as crucial. Improvement could be accomplished even by a modest weight loss; however, the compliance with this form of treatment is often very

S. E. Schiza $(\triangle) \cdot$ C. Mermigkis $\cdot$ I. Bouloukaki

Sleep Disorders Unit, Department of Thoracic Medicine, University

General Hospital, Medical School of the University of Crete,

71110 Heraklion, Crete, Greece

e-mail: schiza@med.uoc.gr low. With this in mind, bariatric surgical procedures according to body mass index (BMI) stratification are preferred, achieving substantial weight loss and far exceeding the effectiveness of non-surgical weight loss programmes. In terms of longterm effectiveness, a recent study showed that patients have achieved and maintained a loss of nearly half of their excess weight for as long as 15 years after bariatric surgery [4].

In this issue of Sleep and Breathing, Dr. Morong and colleagues tried to determine the prevalence of POSA in patients undergoing bariatric surgery and to evaluate the influence of bariatric surgery on POSA. Furthermore, based on previous studies, they choose four predictors for POSA: BMI, neck circumference, AHI and age. The study was performed on 162 patients, and 91 of them were finally analyzed. The authors found that the prevalence of POSA in patients undergoing bariatric surgery was significantly lower (34\%) than the prevalence noted in the general population, and a low AHI was shown to be the only significant independent predictor for the presence of POSA. Their principal finding was that weight loss following bariatric surgery, to a magnitude of $9 \mathrm{~kg} / \mathrm{m}^{2}$ with a concomitant reduction in $\mathrm{AHI}$ about 19 events/h and an improvement in desaturation indices, was related to the transition from non-positional OSA into positional OSA ( $\sim 65 \%$ of patients). Although in retrospective, this study extends previous observations of interaction between changes in body weight, and its association with changes in positional dominance in non-treated patients with OSA [5]. However, the use of a bariatric surgery group in this study, where magnitude of weight loss is greater than in clinical OSA groups, has allowed the authors to state that non-positional OSA can switch to mild positional OSA following weight loss.

Knowledge of positional dependency might be of importance in diagnosis as well as in the evaluation of treatment efficacy of OSA patients. The high prevalence of positional OSA in the less severe forms of OSA have several implications in both clinical practice and research as mild OSA 
patients, which are the vast majority of OSA patients [6] that might be good candidates for positional therapy. Furthermore, severe non-positional OSA patients who cannot adhere to CPAP could use PT after losing weight, allowing them to have an improved quality of sleep and life. On the other hand, POSA patients using successfully PT should be warned that increasing weight may convert them into non-positional OSA who no longer benefit from PT, and therefore, for them, CPAP is the treatment of choice.

Nevertheless, it is worth noting that patients whose OSA improves or resolves after weight loss should strive to maintain their weight loss since weight gain is associated with worsening of OSA. Although data addressing weight loss in OSA patients are somewhat limited, the data available suggest that weight loss can be a highly effective treatment in the short term [7]. Such patients should also be followed closely because OSA may recur even in patients who maintain their weight loss [8]. Because of this, clinicians remain sceptical of the overall efficacy of weight loss in these patients. Counselling regarding ongoing diet modification and exercise, as well as referral to a nutritionist, may be beneficial.

In the literature, an increasing amount of studies has been published on the role of sleep position in OSA and PT. Positional therapy can be defined as preventing patients to sleep in the worst sleeping position, which is usually, but not always, the supine position. When this is the case, forcing a change to the non-supine position during sleep may be an effective treatment. So far, due to the usage of bulky masses placed in the back, compliance with PT was an issue. Poor compliance and the subsequent disappointing long-term results of PT were reported attributed to backache, discomfort and no improvement in sleep quality or daytime alertness. However, various techniques have developed such as positional alarms, verbal instructions, vests, "shark fins" or special pillows, which look promising. Although, the effectiveness of PT in positional OSA has been tested since the 1980s, longterm compliance data and well-powered randomized controlled trials are lacking. In the meta-analysis by $\mathrm{Ha}$ et al. [9], of the only three randomized trials comparing PT vs. continuous positive airway pressure (CPAP), it was found that patients with mild positional OSA may benefit more from PT than those with severe OSA. Furthermore, in this group of patients with mild disease, PT appeared to be almost as effective as CPAP therapy [10]. Thus, PT would appear to be a reasonable choice in patients with a mild disease with a positional component, leading to a powerful therapeutic effect and thus helping avoid the deleterious health and behavioural consequences of OSA.

One could ask why this kind of therapy remains largely ignored and has not been investigated more thoroughly into the daily OSA diagnosis and treatment. Unfortunately, the records of body position changes during all polysomnography (PSG) evaluations, although being mentioned in the PSG report, are not being used further. Furthermore, findings of PSG studies should be interpreted with caution as there are data suggesting that patients spend more time in the supine posture during PSG study compared to home sleep. Therefore, considering that the overall AHI is affected by the relative proportion of each of the different sleeping positions, the severity of OSA may be overestimated in the laboratory settings. In fact, a significant night-to-night AHI variability has been reported. As a consequence, sleep study centres need to recognize that and advise their patients to occupy their natural sleep postures during the PSG study. Likewise, PT, although a minimally invasive treatment modality, is mostly reported in a superficial way or not mentioned at all in the PSG reports.

The main limitation of the study of Morong et al. was the retrospective nature of the study leading to the possibility of underestimation of problems residing in the studied population owing to bias in subject selection and interpretation of the data recall bias. Moreover, as the authors explain, another limitation of this study is that other pathophysiological mechanisms that influence positional dependency, apart from weight change, such as alcohol consumption and sedative use during the study period were not taken into account. In addition, accuracy in recording of sleep posture should be considered, as direct review of videotape recordings of the patient and real-time posture scoring by the nighttime technicians appears to yield more consistent records of sleep posture than that of various digital position monitors. Lastly, in terms of sleep architecture, sleep efficiency improved significantly after bariatric surgery; however, although the patients underwent full polysomnography, there are no information on sleep variables such as arousal index per hour of sleep and percentage of total sleep time spent in each sleep stage.

In conclusion, significant improvement and even remission was noted in obese patients diagnosed with OSA undergoing bariatric surgery. Therefore, clinicians should consider this kind of treatment for patients with severe OSA and obesity alongside with the CPAP. Most of the studies in this area are small, non-randomized, uncontrolled and short term. In order to find out the causality between changes in body weight and its effect on positional OSA, it is imperative to carry out a large, prospective, longitudinal study. Still, positional data are not always used in clinical practice to aid in treatment choices.

\section{References}

1. Oksenberg A, Silverberg DS, Arons E, Radwan H (1997) Positional vs nonpositional obstructive sleep apnea patients. Anthropomorphic, nocturnal polysomnographic and multiple sleep latency test data. Chest 112:629-639

2. Morgenthaler TI, Kapen S, Lee-Chiong T et al (2006) Practice parameters for the medical therapy of obstructive sleep apnea. Sleep 29:1031-1035 
3. Oksenberg A, Khamaysi I, Silverberg DS, Tarasiuk A (2000) Association of body position with severity of apneic events in patients with severe nonpositional obstructive sleep apnea. Chest 118 : 1018-1024

4. O'Brien PE, MacDonald L, Anderson M, Brennan L, Brown WA (2013) Long-term outcomes after bariatric surgery: fifteen-year follow-up of adjustable gastric banding and a systematic review of the bariatric surgical literature. Ann Surg 257:87-94

5. Oksenberg A, Dynia A, Nasser K, Gadoth N (2012) Obstructive sleep apnoea in adults: body postures and weight changes interactions. J Sleep Res 21:402-409

6. Young T, Palta M, Dempsey J, Skatrud J, Weber S, Badr S (1993) The occurrence of sleep-disordered breathing among middle-aged adults. N Engl J Med 328:1230-1235
7. Papandreou C, Schiza SE, Bouloukaki I, Hatzis CM, Kafatos AG, Siafakas NM, Tzanakis NE (2012) Effect of Mediterranean diet versus prudent diet combined with physical activity on OSAS: a randomised trial. Eur Respir J 39:1398-1404

8. Pillar G, Peled R, Lavie P (1994) Recurrence of sleep apnea without concomitant weight increase 7.5 years after weight reduction surgery. Chest 106:1702-1704

9. Ha SC, Hirai HW, Tsoi KK (2014) Comparison of positional therapy versus continuous positive airway pressure in patients with positional obstructive sleep apnea: a meta-analysis of randomized trials. Sleep Med Rev 18:19-24

10. Jokic R, Klimaszewski A, Crossley M, Sridhar G, Fitzpatrick MF (1999) Positional treatment vs continuous positive airway pressure in patients with positional obstructive sleep apnea syndrome. Chest 115: $771-781$ 